\title{
Exploiting the behaviour of wild malaria vectors to achieve high infection with fungal biocontrol agents
}

\author{
Ladslaus L Mnyone ${ }^{1,2,3}$, Issa N Lyimo ${ }^{1,4}$, Dickson W Lwetoijera', Monica W Mpingwa', Nuru Nchimbi', \\ Penelope A Hancock ${ }^{5}$, Tanya L Russell ${ }^{1,6,7}$, Matthew J Kirbyy ${ }^{1,2}$, Willem Takken ${ }^{2}$ and Constantianus JM Koenraadt ${ }^{2 *}$
}

\begin{abstract}
Background: Control of mosquitoes that transmit malaria has been the mainstay in the fight against the disease, but alternative methods are required in view of emerging insecticide resistance. Entomopathogenic fungi are candidate alternatives, but to date, few trials have translated the use of these agents to field-based evaluations of their actual impact on mosquito survival and malaria risk. Mineral oil-formulations of the entomopathogenic fungi Metarhizium anisopliae and Beauveria bassiana were applied using five different techniques that each exploited the behaviour of malaria mosquitoes when entering, host-seeking or resting in experimental huts in a malaria endemic area of rural Tanzania.
\end{abstract}

Results: Survival of mosquitoes was reduced by $39-57 \%$ relative to controls after forcing upward house-entry of mosquitoes through fungus treated baffles attached to the eaves or after application of fungus-treated surfaces around an occupied bed net (bed net strip design). Moreover, 68 to 76\% of the treatment mosquitoes showed fungal growth and thus had sufficient contact with fungus treated surfaces. A population dynamic model of malaria-mosquito interactions shows that these infection rates reduce malaria transmission by $75-80 \%$ due to the effect of fungal infection on adult mortality alone. The model also demonstrated that even if a high proportion of the mosquitoes exhibits outdoor biting behaviour, malaria transmission was still significantly reduced.

Conclusions: Entomopathogenic fungi strongly affect mosquito survival and have a high predicted impact on malaria transmission. These entomopathogens represent a viable alternative for malaria control, especially if they are used as part of an integrated vector management strategy.

\section{Background}

Currently, insecticide treated nets (ITNs) and indoor residual spraying (IRS) are the mainstay of global efforts towards malaria elimination [1,2]. These measures have proven effective in controlling the disease, but this is threatened by the mosquito vectors developing resistance to the synthetic insecticides [3-6]. For example, assessment of the effect of ITNs in Benin revealed that in areas with insecticide-resistant populations of Anopheles gambiae, ITNs no longer prevent such mosquitoes from blood feeding or increase their mortality $[7,8]$. Similarly, in Senegal, a rebound and age shift in malaria

\footnotetext{
* Correspondence: sander.koenraadt@wur.nl

${ }^{2}$ Laboratory of Entomology, Wageningen University and Research Centre, P.

O. Box 8031, $6700 \mathrm{EH}$ Wageningen, The Netherlands

Full list of author information is available at the end of the article
}

cases was observed following introduction of ITNs and artemisinin combination therapy [9]. Clearly, there is an urgent need to develop novel malaria control strategies that can be reliably and sustainably used to complement or replace existing control measures [10]. Biological control of adult mosquitoes using entomopathogenic fungi offers such an alternative approach. Laboratory and small-scale field trials have demonstrated that malaria vectors can succumb to fungus infection [11-15]. Moreover, mosquitoes resistant to insecticides are still vulnerable to fungal infection, and insecticides and fungi could even work synergistically [16-18]. However, efficient techniques that disseminate fungus at larger scale to populations of wild malaria mosquitoes have not been developed [19].

\section{Biomed Central}

() 2012 Mnyone et al; licensee BioMed Central Ltd. This is an Open Access article distributed under the terms of the Creative Commons Attribution License (http://creativecommons.org/licenses/by/2.0), which permits unrestricted use, distribution, and reproduction in any medium, provided the original work is properly cited. 
Host-seeking mosquitoes could be targeted with fungal formulations when entering a house through the eaves $[20,21]$, or when attacking a host under a bed net. Resting mosquitoes could be targeted indoors on walls [22] or both indoors and outdoors by means of point source targets, e.g. resting boxes, clay pots, and black cotton cloths attached to the roof or wall $[12,15,23]$. Outdoor bait-stations have also demonstrated potential as dissemination tools [13]. In reality, the effectiveness of a particular delivery technique will depend on the behaviour of the locally transmitting malaria vector species. For successful control with entomopathogenic fungus it is not only necessary that the mosquito contacts a treated surface, but also receives a sufficient dose of infectious conidia upon this contact $[24,25]$.

The effectiveness of five different techniques of fungal exposure was examined. Each of these exploited the behaviour of mosquitoes when they were either entering, host-seeking or resting in experimental huts in a highly malaria endemic area in rural Tanzania. As African malaria vectors tend to blood feed and rest primarily indoors [26], fungal delivery techniques focused on exposing mosquitoes to entomopathogenic fungi inside the house. Based on the obtained data on fungal infection rates and virulence, a population dynamic model of mosquito-malaria interactions was implemented to estimate the impact of fungal infection on malaria transmission. In addition, the role of outdoor biting behaviour on the effectiveness of the approach was explored.

\section{Methods}

Study area

The field trials were conducted in Lupiro village $\left(8.38^{\circ}\right.$ $\mathrm{S}$ and $36.67^{\circ} \mathrm{E}$ ) (Ulanga District), a rural hamlet 30 $\mathrm{km}$ south of Ifakara, in the Kilombero valley of Tanzania. The village lies on a low plateau of about $10 \mathrm{~m}$ above the surrounding area at an altitude of $300 \mathrm{~m}$ above sea level. The area borders a permanent swamp (near the Ndolo River) extensively cleared for rice cultivation. Most of the residents are farmers and in addition to rice they cultivate maize and cassava. The majority of the houses are made from mud walls with thatched roofs. There are two rainy seasons: the long rains from April to June and short rains normally in October and November. The annual rainfall is about $1200-1800 \mathrm{~mm}$. The temperature ranges between $20^{\circ}$ $\mathrm{C}$ and $32.6{ }^{\circ} \mathrm{C}$. The present study was conducted between May and December 2009 (trials 1-4) as well as between March and April 2010 (trial 5). The population of malaria vectors in the area is largely comprised of members of the An. gambiae complex, mainly Anopheles arabiensis (98\%) and few An. gambiae sensu stricto [27]. Anopheles funestus occurs at low densities. Estimates of the entomological inoculation rate indicate that people receive an estimated 81 infectious bites per year [28].

\section{Entomopathogenic fungus species and formulation}

Two fungal species, Metarhizium anisopliae var. anisopliae (isolates ICIPE-30 and IP 46 [29]) and Beauveria bassiana (isolate: I93-825 (IMI 391510)) were used in the trials. The species and strains of fungus actually used in each trial depended on availability (see Experimental hut trials). Fungal conidia were suspended in a 1: 1 mixture of highly refined Enerpar oil (Enerpar M002 ${ }^{\circledR}$, BP Southern Africa Ltd) and Shellsol oil (Shellsol $\mathrm{T}^{\circledR}$, South Africa Chemicals) [30]. Except for the netting materials in trial 1 (left to dry in the shade for 5 h), cloth materials were left to dry indoors for $72 \mathrm{~h}$ at ambient temperature. Unless stated otherwise, treatment of materials was done at the laboratories of the Ifakara Health Institute (30 km from the study site), and the materials were transported to the field site after drying. Before application in the trial, viability of conidia was confirmed by inoculation on Sabouraud dextrose agar (SDA). The percentage germination of conidia used for the first three trials ranged between $80-85 \%$ and that of the conidia used for the last two trials ranged between $70-75 \%$.

\section{Experimental huts}

The experimental huts were designed to represent local housing [31]. The roof consisted of corrugated iron lined with thatch. The outer walls were constructed from canvas. Inner walls were made of removable panels coated with mud, to simulate local mud walls. The huts were $6.5 \mathrm{~m}$ long, $3.5 \mathrm{~m}$ wide and $2 \mathrm{~m}$ high. The height of each structure measured $2.5 \mathrm{~m}$ at the roof apex. Three huts were used in the trials and each hut had 4 windows all fitted with exit traps (Figure 1A). The huts were positioned between the village and a nearby rice field, standing approximately $15 \mathrm{~m}$ apart. In 2009, temperature and relative humidity inside the experimental huts ranged from $13.8-37.9^{\circ} \mathrm{C}$ and $30.3-100 \% \mathrm{RH}$, respectively. For the experimental months in 2010, temperature and relative humidity ranged from $22.5-37.7^{\circ}$ $\mathrm{C}$ and 45.0 - 99.8\% RH, respectively.

\section{Experimental hut trials}

Three experimental huts were used in each of five trials. Each individual trial was conducted for nine days. Unless stated otherwise, a $3 \times 3$ Latin square design was used to simultaneously evaluate two different designs for delivering entomopathogenic fungi and one control. Treatments were randomly allocated to the three different huts, and were switched between huts each time after three days. During the trials each hut contained two human volunteers sleeping under untreated bed 

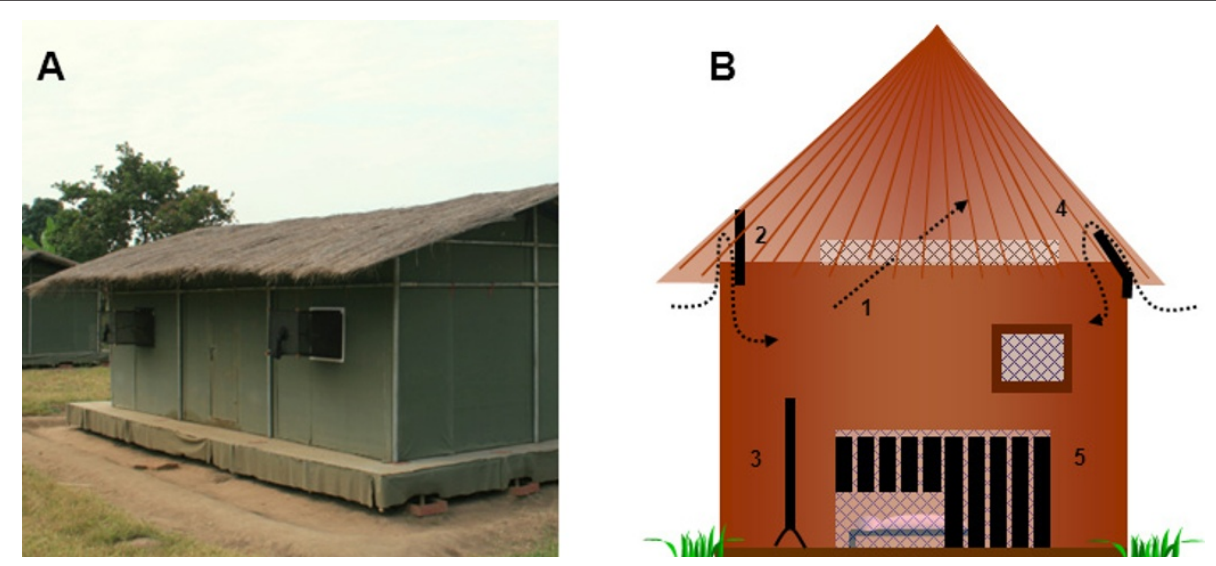

Figure 1 A: picture of experimental hut in Lupiro, Tanzania; B: schematic drawing of the five designs evaluated for infecting malaria vectors with entomopathogenic fungi inside the experimental huts. 1: eave netting, 2: cotton cloth eave curtain, 3: cotton cloth panel, 4: cotton cloth eave baffle and 5: cotton cloth strips around bed net.

nets. The volunteers rotated between huts daily such that each pair spent three days in each hut in total. The volunteers slept in the experimental huts from 19.00 $7.00 \mathrm{~h}$. Exit traps were installed over all four windows (trap: $55.5 \times 45.5 \times 55.5 \mathrm{~cm}$; funnel opening: $40 \times 3 \mathrm{~cm}$ ) to catch mosquitoes when they exit, except for trial 1 where two window and six eave traps (trap: $100 \times 40 \times$ $40 \mathrm{~cm}$; funnel opening: $80 \times 3 \mathrm{~cm}$ ) were used. Mosquitoes were collected by mouth aspirator from the exit traps at $7.00 \mathrm{am}$ in the morning. A random sub-sample of maximum 25 female mosquitoes from each hut was collected. Each mosquito was placed individually into 50 $\mathrm{ml}$ falcon tubes $(115 \mathrm{~mm} \times 28 \mathrm{~mm}$ diam.) that were covered with gauze and provided with sugar watersoaked cotton-wool on top. Daily survival of mosquitoes was monitored in a field-based insectary until all mosquitoes had died (maximum time to death: 39 days). When less than 25 mosquitoes were trapped in the window exit traps, all available female individuals were used. Mosquitoes remaining in the traps were killed, identified and stored. Cadavers of mosquitoes that died in the survival experiment were left to dry in open air for 2 day, put onto filter paper in Petri dishes covered with their lids and kept inside transparent containers (30 cm diameter and $40 \mathrm{~cm}$ high) with wet towels (humid chambers) for 5-6 day. Thereafter, they were examined for fungal sporulation. Containers were tightly closed with lids to maintain humidity.

\section{Trial 1: Eave netting}

Fungus-treated polyester netting $\left(9\right.$ holes $\left./ \mathrm{cm}^{2}\right)$ was fitted over the eaves of the experimental huts to determine whether wild mosquitoes could be infected during passage through this netting (Figure 1-B). In the laboratory, $40 \%$ of the mosquitoes passed through this type of netting when exposed to a human stimulus. A fungal formulation of Beauveria bassiana 193-825 was painted with a brush onto the netting laid on a flat surface at a concentration of $2 \times 10^{10}$ conidia $/ \mathrm{m}^{2}$. Treated netting was left outdoors in the shade to dry for about $5 \mathrm{~h}$, before being fitted over the eaves with Velcro strips. Two controls were used: eaves with oil-treated netting, and eaves without netting.

\section{Trial 2: Eave curtain}

Black cotton curtains $(20 \mathrm{~cm}$ high) were fixed from the top with Velcro strips and left hanging in front of the eaves, leaving a gap of about $3.5 \mathrm{~cm}$ between the cloth and the wall (Figure 1B). Mosquitoes passing the eaves could fly into the hut through the gap at the lower end of the curtain, probably after contacting the curtain to locate the gap. One hut had curtain treated with oil alone (control), and the two other huts had curtains treated with B. bassiana I93-825 or M. anisopliae ICIPE-30, respectively. Both isolates were applied onto curtains at a concentration of $2 \times 10^{10}$ conidia $/ \mathrm{m}^{2}$ using procedures described by Mnyone et al. [14]. Only the surface of the curtain facing the outside was treated.

\section{Trial 3: Eave curtains and panels}

Eave curtains were placed in one of the huts, as in Trial 2 , except that a four times higher concentration of fungus $\left(8 \times 10^{10}\right.$ conidia $\left./ \mathrm{m}^{2}\right)$ was used. In the other hut, two black cotton panels (length $120 \mathrm{~cm}$, width $90 \mathrm{~cm}$ ), one per bed were used. Panels were treated with fungus on both sides by the use of a hand sprayer [14], and were placed next to the bed facing the feet of a sleeping volunteer (Figure 1B). Curtains and panels were both treated with $B$. bassiana. The control hut had neither curtain nor panel and eaves were left open. 


\section{Trial 4: Eave baffles}

Eave curtains were modified to serve as eave baffles [32]. Unlike curtains that were parallel to the wall and had the entry gap at the lower side, cotton cloth baffles were fitted in a slanting orientation with the entry gap $(3 \mathrm{~cm})$ from the top ( $\approx 20 \mathrm{~cm}$ inside the wall) (Figure $1 \mathrm{~B}$ ). At the bottom, the cloth material was fixed with pins to the outside wall covering $\approx 10 \mathrm{~cm}$ of the outside wall surface. One hut had baffles treated with Beauveria bassiana 193-825 and one had baffles treated with $M$. anisopliae IP 46 at a concentration of $4.1 \times 10^{10}$ coni$\mathrm{dia} / \mathrm{m}^{2}$. The control hut had eave baffles treated with oil alone.

\section{Trial 5: Bed net strips}

Long black cotton strips $(126 \mathrm{~cm}, 7 \mathrm{~cm}$ wide) and short black cotton strips $(63 \mathrm{~cm}, 7 \mathrm{~cm}$ wide) were treated on one side with $M$. anisopliae IP 46 at a concentration of $5 \times 10^{10}$ conidia $\mathrm{m}^{-2}$ and installed next to bed nets to surround the entire bed (Figure 1). The gap between the strips was $1 \mathrm{~cm}$. One hut had short cloth strips, one hut long cloth strips and the third hut did not have any strips (control). Treatment of the strips was done at the field site at 17: $00 \mathrm{~h}$ to minimize the effect of sun light on conidia when drying. Strips were left outdoors for 3 $\mathrm{h}$ to allow initial drying, then installed inside huts and left for an extra $24 \mathrm{~h}$ to complete drying.

\section{Data analysis and modelling}

Weibull functions representing proportional survival of adult mosquitoes as a function of time were fitted to the survival data of the daily-collected mosquitoes from the experimental huts (see Additional file 1 and [33]). These functions were used to estimate the average time to death for mosquitoes collected from control and treatment huts for each trial. These estimates, along with the observed daily fungal infection rates, were used to parameterize a population dynamic model developed by of one of us (PAH) [33] to estimate the impact of fungus application on malaria transmission rate, expressed as the daily entomological inoculation rate (EIR) [34]. The model incorporates details of mosquito life history relevant to the effect of fungal biopesticide interventions on malaria transmission, including time-dependent adult mortality, gonotrophic feeding processes (host-seeking and non-host-seeking mosquitoes) and insecticide resistance. Unless otherwise specified the same parameter values as those in Hancock (2009) were used [33].

The coverage of the biopesticide was defined as the daily probability that host-seeking mosquitoes contract the fungus, and assume that non-host-seeking mosquitoes are not at risk of fungal infection [33]. The fitted Weibull functions were used to estimate the effect of fungal infection on adult mosquito mortality in the absence of all other mortality sources (see Additional file 1). The average time until death from fungal infection excluding all other mortality sources is used to summarize the effect of infection on mosquito mortality and can be considered a measure of the 'virulence' of the fungal biopesticide.

\section{Modelling the impact of biting behaviour}

The model also allows for the incorporation of fine-scale temporal variation in biting behaviour. The goal of this exercise was to account for outdoor biting patterns of An. arabiensis populations at the study site ([35]). Given that data are not available to accurately quantify biting behaviour and its implications for fungal infection risk, a conservative set of assumptions are adopted. Parameters relating to indoor and outdoor biting behaviour are given in Table 1 and a more detailed explanation of the full model and its parameterization is given in Additional file 2. In summary, the mosquito population is divided into two subpopulations that display different levels of indoor and outdoor biting behaviour. The first subpopulation is endophilic and seeks blood meals indoors when humans are indoors. The fungal infection risk for this subpopulation is parameterized using data from the experimental hut trials. The second subpopulation is exophilic and seeks hosts outdoors for most of the time that humans are indoors. Both subpopulations seek human blood meals during times that humans are outdoors, during which time mosquitoes are assumed to be not exposed to the biopesticide. The endophilic subpopulation takes blood meals only from humans while the exophilic subpopulation may take human or nonhuman blood meals. Therefore, while the endophilic subpopulation is responsible for greater levels of malaria transmission, it is also exposed to a greater risk of fungal infection.

\section{Ethical approval}

The study was conducted after being approved by the Institutional Review Board of the Ifakara Health Institute (IHI) (IHRDC/IRB/No. A-019) and the National Institute of Medical Research (NIMR/HQ/R.8a/Vol. IX/ 710) in Tanzania.

\section{Results \\ Experimental hut trials}

The netting prevented females from entering, but failed to infect them with fungus (trial 1; Table 2). Treated eave curtains and cloth panels also failed to infect mosquitoes with either M. anisopliae or B. bassiana. Although the increased dosage in trial 3 resulted in $11-18 \%$ of mosquitoes being infected with $B$. bassi$a n a$, exposure to entomopathogenic fungi only resulted in slightly elevated mortality (trials 2 and 3; Table 2; 
Table 1 Parameter definitions and assumed values of the model of Hancock et al.

\begin{tabular}{|c|c|c|c|}
\hline Symbol & Definition & Value & Source \\
\hline$f$ & Rate of finding blood meals for host-seeking mosquitoes & $2.4\left(d^{-1}\right)$ & [36] \\
\hline$P$ & $\begin{array}{l}\text { Probability of finding a blood meal for host-seeking mosquitoes within a } 12 \text { hour host-seeking period, given that } \\
\text { they do not die }\left(1-\exp \left(-T_{H} f\right)\right)\end{array}$ & 0.7 & \\
\hline$p_{E}$ & Proportion of the total mosquito population that comprise the exophillic subpopulation & 0.58 & [35] \\
\hline Q & Probability that exophillic mosquitoes feed on a human host & $1 / 3$ & [37] \\
\hline$T_{H}$ & Duration of the host-seeking period & $0.5(d)$ & [35] \\
\hline po & Proportion of the host-seeking period during which humans are outdoors & 0.2 & [35] \\
\hline F & Rate of contracting fungal infection for endophilic and exophilic subpopulations & $\begin{array}{l}3.0,3.0 / 5 \\
\left(d^{-1}\right)\end{array}$ & $\begin{array}{l}\text { This } \\
\text { study }\end{array}$ \\
\hline$C$ & $\begin{array}{l}\text { Probability of fungal infection during the period of biopesticide exposure for endophilic and exophilic } \\
\text { subpopulations }\left(1-\exp \left(-\left(1-p_{o}\right) T_{H} F\right)\right)\end{array}$ & $0.7,0.2$ & $\begin{array}{l}\text { This } \\
\text { study }\end{array}$ \\
\hline
\end{tabular}

[34]

see Additional files 3 and 4 for estimated model parameters).

In trial 4 (eave baffles), the average number of female An. gambiae mosquitoes collected per hut per night ranged from 40.6 to 74.2 (Table 2). Of 178 mosquitoes that were sampled from the $M$. anisopliae IP 46 treated hut, 69\% showed fungal growth. For the B. bassiana treated hut, $68 \%$ of 206 mosquitoes showed fungal growth (Table 2). Of the controls, $2.0 \%$ showed fungal growth. Mosquitoes from huts with fungus-treated baffles survived less long relative to mosquitoes collected from the control hut, regardless of the fungal species (Table 2; Figure 2-A; see Additional files 5 and 6 for estimated model parameters). Average time to death of fungus-infected mosquitoes was reduced by $43 \%$ ( $M$. anisopliae) and $38 \%$ (B. bassiana) compared to mosquitoes collected from control huts. It was reduced by $44 \%$ (M. anisopliae) and $41 \%$ (B. bassiana) compared to uninfected females collected from the same treatment.

In trial 5 (bed net strips), the average number of female An. gambiae mosquitoes collected per hut per night ranged from 24.3 to 86.2 (Table 2). Of 155 mosquitoes sampled from huts with fungus-treated long strips, $75.5 \%$ showed fungal growth. From huts with treated short strips, $74.6 \%$ of 189 mosquitoes showed fungal growth. Of the controls, 3.3\% showed growth of M. anisopliae (Table 2). Survival of mosquitoes from huts with both long and short strips was substantially

Table 2 Number of female Anopheles gambiae s.l. collected nightly from each treatment of the five experimental hut trials, the proportion that showed fungal growth after death (Sporulation \%) and the average time to death (g) derived from the Weibull models in days (see Additional file 1)

\begin{tabular}{|c|c|c|c|c|c|c|c|c|}
\hline \multirow[b]{2}{*}{ Trial } & \multirow[b]{2}{*}{ Design } & \multirow[b]{2}{*}{ Treatment } & \multirow[b]{2}{*}{ Dosage (conidia/m²) } & \multirow[b]{2}{*}{ Average catch per night } & \multirow[b]{2}{*}{ Sporulation (\%) } & \multicolumn{3}{|c|}{ Avg. time to death $(g)$} \\
\hline & & & & & & All & Uninfected & Infected \\
\hline \multirow[t]{3}{*}{1} & Eave netting & Bb 193-825 & $2 * 10^{10}$ & $1.1 \pm 0.35$ & 0.0 & nd & & \\
\hline & & Oil control & & $1.0 \pm 0.24$ & 0.0 & nd & & \\
\hline & & Open eaves (control) & & $45.0 \pm 7.8$ & 0.0 & nd & & \\
\hline \multirow[t]{3}{*}{2} & Eave curtain & Ma ICIPE-30 & $2 * 10^{10}$ & $36.4 \pm 6.0$ & 0.0 & 15.6 & & \\
\hline & & Bb $193-825$ & $2 * 10^{10}$ & $32.4 \pm 4.4$ & 0.0 & 17.2 & & \\
\hline & & Oil control & & $38.4 \pm 4.1$ & 0.0 & 18.4 & & \\
\hline \multirow[t]{3}{*}{3} & Eave curtain & Bb 193-825 on curtain & $8^{*} 10^{10}$ & $27.9 \pm 1.7$ & 18.3 & 18.5 & & \\
\hline & \& panels & Bb 193-825 on panels & $8^{*} 10^{10}$ & $30.2 \pm 1.3$ & 10.7 & 17.3 & & \\
\hline & & Open eaves (control) & & $33.9 \pm 1.2$ & 0.0 & 20.6 & & \\
\hline \multirow[t]{3}{*}{4} & Eave baffles & Ma IP 46 & $4.1 * 10^{10}$ & $40.6 \pm 8$ & 69.1 & & 20.7 & 11.5 \\
\hline & & Bb $193-825$ & $4.1 * 10^{10}$ & $56.3 \pm 11.4$ & 67.9 & & 17.9 & 10.5 \\
\hline & & Oil control & & $74.2 \pm 14.5$ & 2.0 & & 20.3 & \\
\hline \multirow[t]{3}{*}{5} & Bed net strips & Ma IP 46 - long & $5^{*} 10^{10}$ & $24.3 \pm 3.2$ & 75.5 & & 24.5 & 10.5 \\
\hline & & Ma IP 46 - short & $5^{*} 10^{10}$ & $38.4 \pm 6.8$ & 74.6 & & 23.0 & 11.8 \\
\hline & & Open eaves (control) & & $86.2 \pm 12.7$ & 3.3 & & 19.2 & \\
\hline
\end{tabular}

As sporulation rates in the trials 2 and 3 were zero or very low, we only calculated separate 'average time to death' for fungus-infected individuals (those that sporulated) and fungus-uninfected individuals (those that did not sporulate) of trials 4 and 5 . In trial 1, the number of mosquitoes collected in exit traps of treatment huts was too low to calculate 'average time to death' ('nd' in Table). Ma: Metarhizium anisopliae; Bb: Beauveria bassiana 

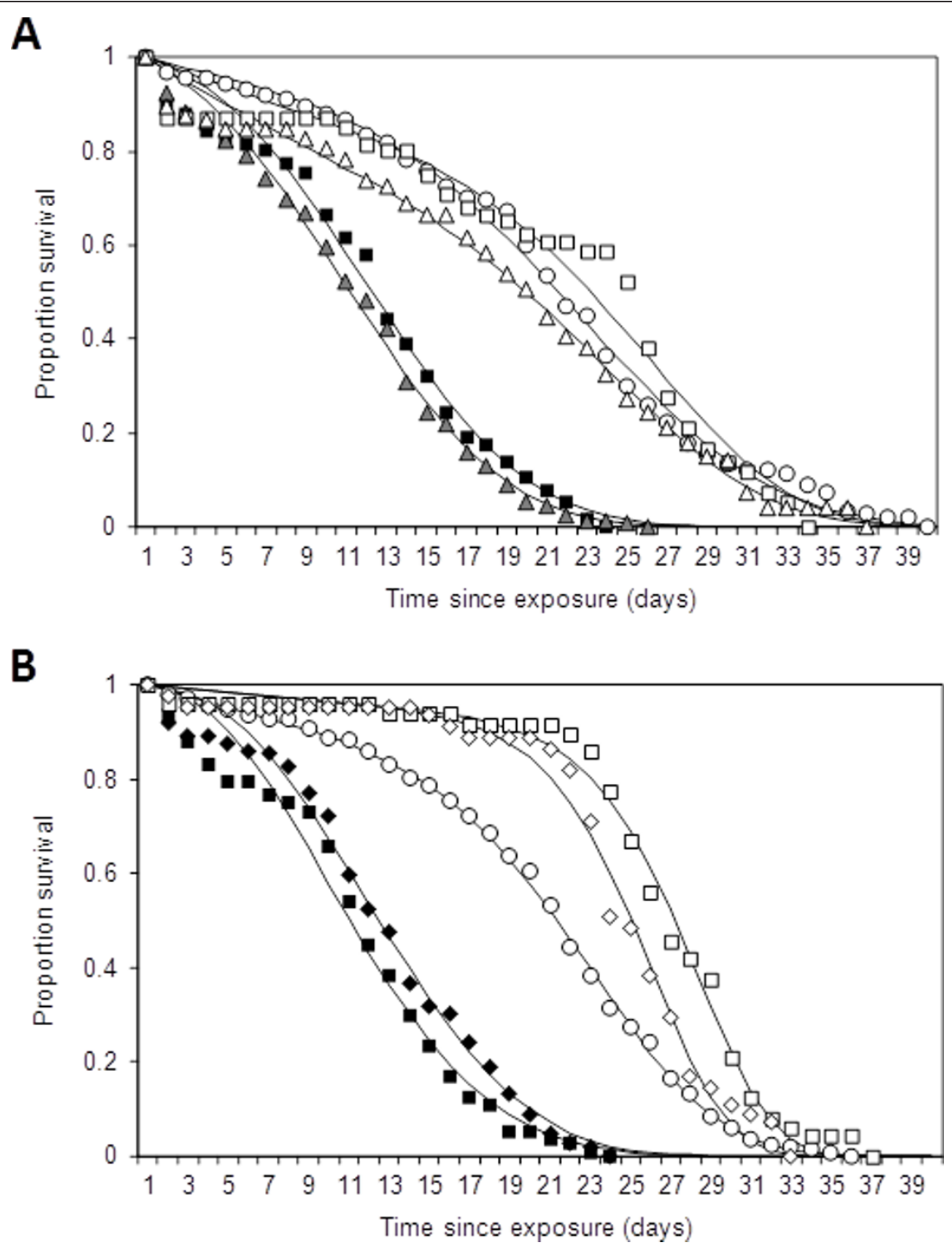

Figure 2 Survival of wild female Anopheles gambiae s.l. mosquitoes collected from experimental huts. A: Results from trial 4 (eave baffles) with white circles: control; white squares: uninfected females from Metarhizium anisopliae IP 46 treated huts; black squares: infected females from M. anisopliae IP 46 treated huts; white triangles: uninfected females from Beauveria bassiana 193-825 treated huts; gray triangles: infected females from B. bassiana 193-825 treated huts. B: Results from trial 5 (cloth strips treated with M. anisopliae IP-46 around bed net) with white circles: control; white diamonds: uninfected females from short strips in the treated hut, black diamonds: infected females from short strips in treated hut; white squares: uninfected females from long strips in the treated hut; black squares: infected females from long strips in the treated hut. Solid lines show Weibull functions fitted to each survival profile using the least squares method (see Additional file 1).

lower compared to mosquitoes collected from the control (Table 2; Figure 2-B; see Additional files 7 and 8 for estimated model parameters): average time to death of fungus-infected mosquitoes was reduced by $45 \%$ (long strips) and 39\% (short strips) compared to mosquitoes collected from control huts. It was reduced by $57 \%$ and $49 \%$, respectively, if compared to uninfected females collected from the same treatment hut.

\section{Impact of entomopathogenic fungi on EIR}

Assuming the most successful delivery methods are employed (eave baffles and bed net strips; trials 4 and 5), a similar impact of M. anisopliae and B. bassiana on malaria transmission can be expected because the experiments using both isolates produced similar reductions in survival (Figure 2). Therefore, only the survival data of mosquitoes exposed to B. bassiana applied on 
eave baffles (trial 4; Figure 2A) were used to estimate the parameters of the Weibull function describing the time-dependent effect of fungal infection on mosquito mortality. Based on fungal infection rates obtained from the experiment, the daily probability of fungal infection for host-seeking mosquitoes, or the fungal coverage, is estimated to be 0.68 . For these levels of coverage and effects on mortality, the estimated reduction in the daily EIR that could be achieved by our B. bassiana application is $75-80 \%$ (Figure 3).

\section{Impact of feeding behaviour}

With a fungus coverage level as observed in the present study (0.7), the efficacy of the fungus is not greatly reduced when approximately $58 \%$ of the total population displays exophilic biting behaviour (Russell et al., 2011, Figure 4). Similar conclusions can be drawn when coverage levels are lower, provided that the coverage is not at very low levels e.g. below 0.2 (see Additional file 9). In general, only when the whole population starts displaying exophilic behaviour, the effectiveness of the indoor intervention is substantially reduced.

\section{Discussion}

Up to three quarters of house-entering mosquitoes became infected with entomopathogenic fungi when either the eaves were provided with fungus-treated baffles or when strips of fungus-treated cotton cloth were hung around the bed net. This infection resulted in an increased daily risk of death and, according to the model, such coverage and virulence rates lead to an estimated $75-80 \%$ reduction in malaria transmission. By contrast, eave netting, eave curtains and cotton panels placed next to the bed were ineffective in exposing mosquitoes to fungi and did not affect survival or even infect mosquitoes with fungal spores. Mosquitoes which contacted fungus-treated netting (trial 1) and flew off may have become infected, but this could not be

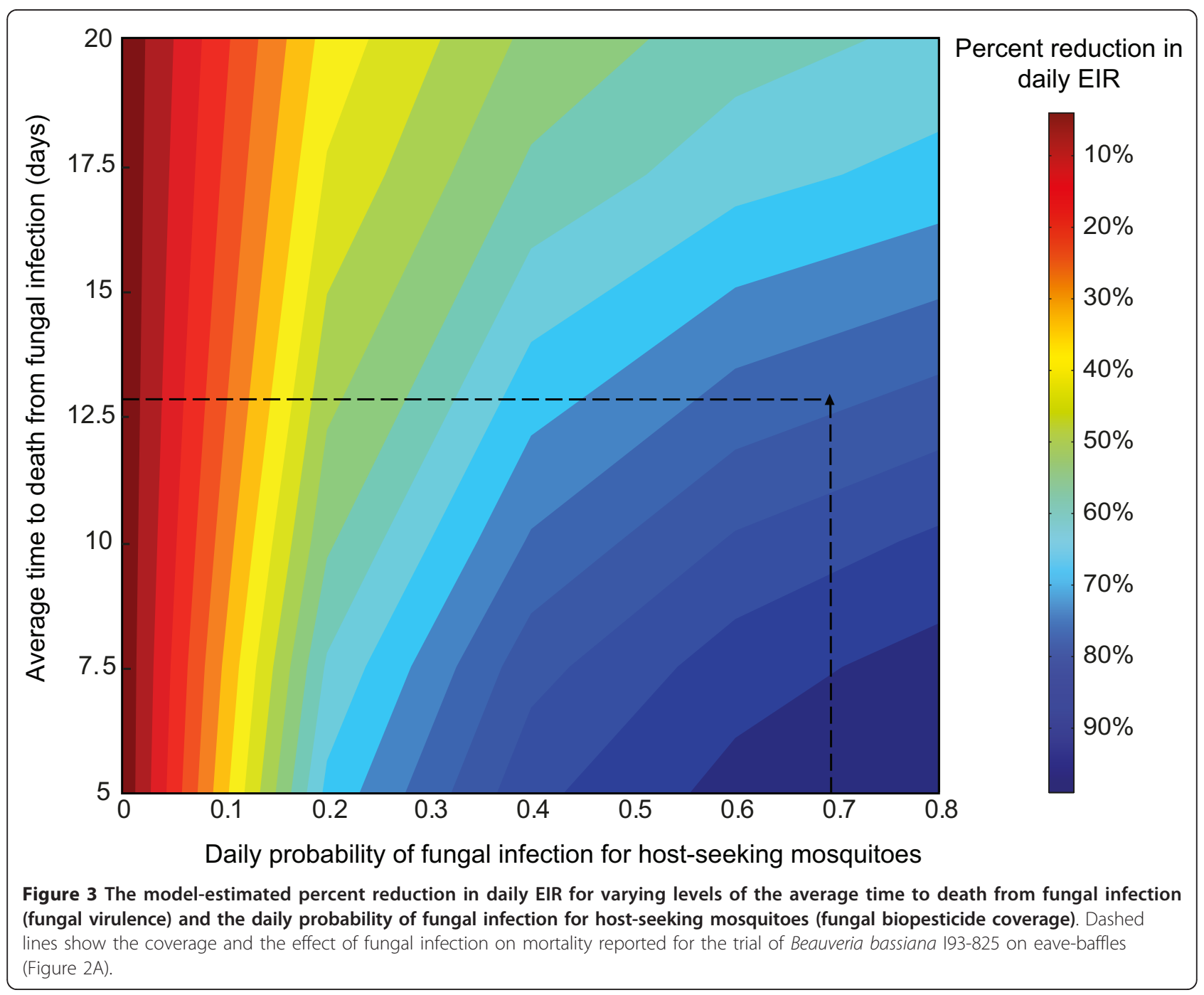




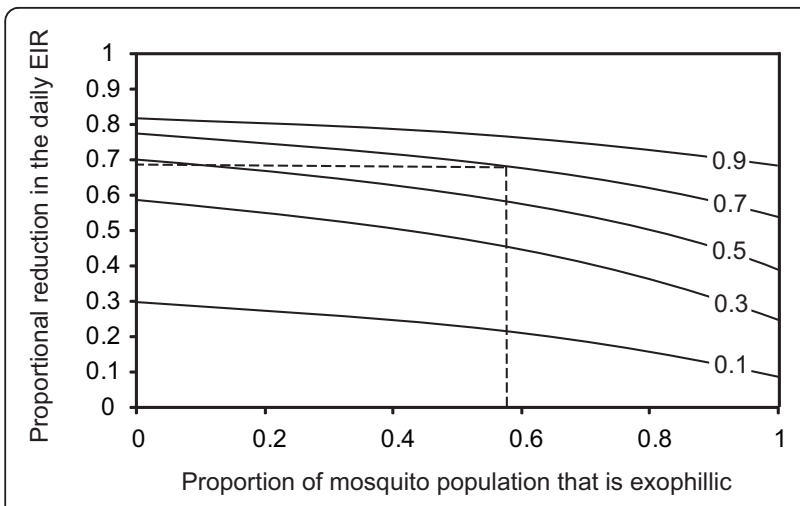

Figure 4 The proportional reduction in the daily EIR as a function of the proportion of the mosquito population that is exophilic. Line labels show the fungal biopesticide coverage. Dotted lines show the reduction in EIR for the biopesticide coverage value measured in the experimental hut trials (this study) and the estimated proportion of the An. arabiensis population that is exophilic for the study area [35].

confirmed with the experimental design that was used. Conidia could lose their potency rapidly when applied on netting $[38,39]$. With eave curtains made of cotton cloth (trial 2), mosquitoes possibly flew directly into the huts without contacting the treated surfaces or spent too little time on the cloth to pick up conidia [24,25]. This may result in small infective doses that can be countered by immune responses such as melanisation, encapsulation and phagocytosis [40]. Although the concentration of fungal conidia in the subsequent trial (trial 3) was four times higher, the impact of the fungus was still small with fungus infection rates reaching 11 and $18 \%$ for curtains and panels, respectively.

Quite strikingly, in huts with baffles that were fitted in a slanted orientation with the entry gap at the top (trial 4), mosquito survival was almost halved. Apparently, a minor modification of the position of fungus-treated surfaces greatly increased the probability of mosquitoes contacting, and spending time on treated surfaces. Similarly, after entering the house, the majority of host-seeking mosquitoes could be targeted when approaching a bed net as demonstrated with the cotton cloth strips hung next to the net. For such fungal tools to have a strong effect on the EIR, it has been estimated that > $50 \%$ of house entering mosquitoes would need to be infected and their survival reduced below the critical age at which they can pass on the malaria parasite $[15,41]$; in the present study a fungal coverage as high as $75 \%$ was achieved. With this coverage level, a more virulent fungus (i.e. with lower time to death) will only marginally increase effectiveness in terms of a further reduction in EIR (see Figure 3; moving down the vertical dashed line). When only a low proportion of the mosquito population comes into contact with the fungus, virulence becomes more important. For example, at a coverage level of 0.3 and a virulence of 12.5 days, the reduction in EIR is $\sim 55 \%$. A virulence of $\sim 6$ days would be required to achieve a similar reduction of $80 \%$ in EIR. Below coverage levels of 0.2 , even highly virulent strains will not achieve major reductions in transmission. Similarly, with the currently achieved coverage, even fungus with relatively low virulence (slow kill), e.g. as a result of natural degradation, can still produce substantial reductions in the EIR (see Figure 3; moving up the vertical dashed line).

The first model estimates presented in this paper are based on the numbers of (mostly An. arabiensis) mosquitoes actually in search for a human host sleeping inside the experimental huts at night. This is the time and location where most of the transmission takes place. However, because ITNs may select for outdoorbiting behaviour within or between species $[35,42]$, the impact of exophily on the effectiveness of the biopesticide approach was explored as well. This model analysis showed that the presence of considerable outdoor feeding activity does not significantly impact on the ability of indoor biopesticide interventions to reduce the EIR. A similar conclusion was reached by modelling analysis which explored the impact of outdoor biting behaviour on the efficacy of indoor ITN interventions [43]. One way outdoor feeding could be addressed is by also targeting these populations with entomopathogenic fungi, e.g. through the use of fungus treated resting stations [13].

Surprisingly, a small proportion $(\leq 3 \%)$ of mosquitoes from control huts showed fungal growth (trials 4 and 5). This could be explained by assuming that few mosquitoes remained undetected in the huts during the removal of mosquitoes prior to switching the treatments or that mosquitoes from treatment huts visited control huts during the same night or later. In trial 4 and 5, the average number of female anopheline mosquitoes collected from control huts was higher than that collected from treatment huts. As treatments and volunteers were rotated among huts, this cannot have been due to a positional effect or individual variation in attractiveness. This difference could be due to a behavioural effect of $M$. anisopliae IP 46, causing repellency or avoidance, but such effects have not been observed with $M$. anisopliae ICIPE-30 and B. bassiana I93-825 in laboratory studies [44]. The behaviour of mosquitoes once infected with a fungus, the sub-lethal consequences of fungal infection [45] and the potential repellency of fungus treated material need to be further investigated under field conditions.

The effectiveness of the bed net strip design demonstrates that it is feasible to develop mosquito control strategies based on lure and kill principles. In the case 
presented here, the protected sleeper acts as the lure, while the strips delivered the lethal doses of entomopathogenic fungi. Because of their focal nature, such strategies are likely to be more cost-effective than residual application of fungus formulations. In addition, entomopathogenic fungi are slow-killing agents, but kill the mosquito before they are capable of transmitting the infective sporozoite stages. This reduces the pressure for selection of resistance, making these agents 'evolutionproof' and thereby enhancing their long-term use [46].

\section{Conclusions}

The results obtained in realistic field settings provide a necessary stepping stone towards scaling up of fungal biocontrol agents to whole village application. They represent a viable alternative for malaria control, especially if they are used as part of an integrated vector management strategy. Efforts geared at producing high quality fungal products in terms of virulence and persistence should be continued as there is an extra benefit to be accrued in terms of their impact on malaria transmission and the sustainability of malaria control programmes.

\section{Additional material}

Additional file 1: Explanation of the methods developed by

Hancock et al. (2009) to model adult mosquito survival and evaluate the impact of entomopathogenic fungi on mosquito mortality and malaria transmission.

Additional file 2: Explanation of assumptions in relation to the model that assesses the effect of outdoor feeding on the efficacy of indoor-based fungal applications on EIR.

Additional file 3: Table S1 Parameters of the model of mosquito mortality estimated from experimental data of trial 2: curtains treated with Beauveria bassiana and Metarhizium anisopliae.

Parameter values were chosen to minimise the residual sum of squares. $\mu$ is the mortality rate (per day), $\beta_{s}$ and $r_{s}$ are the dimensionless shape and rate shape parameters of the Weibull function, respectively, and $g$ is the average time to death (in days) estimated from the Weibull function (see Additional file 1).

Additional file 4: Table S2 Parameters of the model of mosquito mortality estimated from experimental data of trial 3: eave curtains and panels treated with Beauveria bassiana. Parameter values were chosen to minimise the residual sum of squares. $\mu$ is the mortality rate (per day), $\beta_{s}$ and $r_{s}$ are the dimensionless shape and rate shape parameters of the Weibull function, respectively, and $g$ is the average time to death (in days) estimated from the Weibull function (see Additional file 1).

Additional file 5: Table S3 Parameters of the model of mosquito mortality estimated from experimental data of trial 4: eave baffles treated with Metarhizium anisopliae. Parameter values were chosen to minimise the residual sum of squares. Separate models were fitted for mosquitoes infected and uninfected with the fungus. $\mu$ is the mortality rate (per day), $\beta, \beta_{s}$ and $r_{,} r_{s}$ are the dimensionless shape and rate shape parameters of the Weibull functions, respectively. $g$ is the average time to death (in days) estimated from the Weibull function and $g_{F}$ is the estimated average time to death from the fungus infection alone (see Additional file 1).

Additional file 6: Table S4 Parameters of the model of mosquito mortality estimated from experimental data of trial 4: eave baffles treated with Beauveria bassiana. Parameter values were chosen to minimise the residual sum of squares. Separate models were fitted for mosquitoes infected and uninfected with the fungus. Separate models were fitted for mosquitoes infected and uninfected with the fungus. $\mu$ is the mortality rate (per day), $\boldsymbol{\beta}, \boldsymbol{\beta}_{s}$ and $r, r_{s}$ are the dimensionless shape and rate shape parameters of the Weibull functions, respectively. $g$ is the average time to death (in days) estimated from the Weibull function and $g_{F}$ is the estimated average time to death from the fungus infection alone (see Additional file 1).

Additional file 7: Table S5 Parameters of the model of mosquito mortality estimated from experimental data of trial 5: long strips treated with Metarhizium anisopliae. Parameter values were chosen to minimise the residual sum of squares. Separate models were fitted for mosquitoes infected and uninfected with the fungus. Separate models were fitted for mosquitoes infected and uninfected with the fungus. $\mu$ is the mortality rate (per day), $\beta, \beta_{s}$ and $r, r_{s}$ are the dimensionless shape and rate shape parameters of the Weibull functions, respectively. $g$ is the average time to death (in days) estimated from the Weibull function and $g_{F}$ is the estimated average time to death from the fungus infection alone (see Additional file 1).

Additional file 8: Table S6 Parameters of the model of mosquito mortality estimated from experimental data of trial 5: short strips treated with Metarhizium anisopliae. Parameter values were chosen to minimise the residual sum of squares. Separate models were fitted for mosquitoes infected and uninfected with the fungus. Separate models were fitted for mosquitoes infected and uninfected with the fungus. $\mu$ is the mortality rate (per day), $\beta, \beta_{s}$ and $r, r_{s}$ are the dimensionless shape and rate shape parameters of the Weibull functions, respectively. $g$ is the average time to death (in days) estimated from the Weibull function and $g_{F}$ is the estimated average time to death from the fungus infection alone (see Additional file 1).

Additional file 9: Daily EIR as a function of the fungal biopesticide coverage. Line labels show the proportion of the mosquito population that is exophilic.

\section{Acknowledgements}

Financial support was provided by the Adessium Foundation (Reeuwijk, The Netherlands). Beauveria bassiana was kindly provided by Wageningen University, The Netherlands (courtesy M. Jumbe) and Penn State University, USA (courtesy M. Thomas \& N. Jenkins). We thank all those persons who voluntarily slept in the huts during the study. We also thank Novatus Lipindi, Haji Kambwili and Petro Komba for technical assistance and Jason Moore for assisting with the construction of the experimental huts.

\section{Author details}

${ }^{1}$ Biomedical and Environmental Group, Ifakara Health Institute, P.O. Box 53, Off Mlabani Passage, Ifakara, Tanzania. 'Laboratory of Entomology, Wageningen University and Research Centre, P.O. Box 8031, $6700 \mathrm{EH}$ Wageningen, The Netherlands. ${ }^{3}$ Pest Management Centre, Sokoine University of Agriculture, P.O. Box 3110, Morogoro, Tanzania. ${ }^{4}$ Faculty of Biomedical and Life Sciences, University of Glasgow, 120 University Place, G12 8TA Glasgow, UK. ${ }^{5}$ School of Life Sciences, University of Warwick, Coventry CV4 7AL, UK. ${ }^{6}$ The University of Queensland, School of Population Health, Australian Centre for Tropical and International Health, Brisbane 4006, Australia. ${ }^{7}$ Vector Group, Liverpool School of Tropical Medicine, Liverpool L3 5QA, UK.

\section{Authors' contributions}

Conceived and designed the experiments: LLM, INL, MJK, WT. Performed the experiments: LLM, MWM, NN, INL, DWL. Analysed the data: LLM, CJMK, INL. Performed the modelling: PAH. Wrote the paper: LLM, CJMK, WT, TLR. Reviewed the paper: CJMK, MJK, WT. All authors read and approved the final manuscript.

\section{Competing interests}

All authors have no conflicts of interest to declare and all have actively contributed to this study and review. 
Received: 28 February 2012 Accepted: 26 March 2012

Published: 26 March 2012

\section{References}

1. Alonso PL, Besansky NJ, Burkot TR, Collins FH, Hemingway J, James AA, Lengeler C, Lindsay S, Liu Q, Lobo NF, Mnzava A, Tanner M, Zwiebel L: A research agenda for malaria eradication: vector control. PLoS Med 2011, 8:e1000401.

2. Baum J, Billker O, Bousema T, Dinglasan R, McGovern V, Mota MM, Mueller I, Sinden R: A research agenda for malaria eradication: basic science and enabling technologies. PLoS Med 2011, 8:e1000399.

3. Kelly-Hope L, Ranson H, Hemingway J: Lessons from the past: managing insecticide resistance in malaria control and eradication programmes. Lancet Infect Dis 2008, 8:387-389.

4. Ranson H, Abdallah H, Badolo A, Guelbeogo WM, Kerah-Hinzoumbe C, Yangalbe-Kalnone E, Sagnon N, Simard F, Coetzee M: Insecticide resistance in Anopheles gambiae: data from the first year of a multi-country study highlight the extent of the problem. Malar J 2009, 8:299.

5. Ranson H, N'Guessan R, Lines J, Moiroux N, Nkuni Z, Corbel V: Pyrethroid resistance in African anopheline mosquitoes: what are the implications for malaria control? Trends Parasitol 2011, 27:91-98.

6. Butler D: Mosquitoes score in chemical war. Nature 2011, 475:19.

7. N'Guessan R, Corbel V, Akogbeto M, Rowland M: Reduced efficacy of insecticide-treated nets and indoor residual spraying for malaria control in pyrethroid resistance area, Benin. Emerg Infect Dis 2007, 13:199-206.

8. Yadouleton AW, Padonou G, Asidi A, Moiroux N, Bio-Banganna S, Corbel V, N'guessan R, Gbenou D, Yacoubou I, Gazard K, Akogbeto MC: Insecticide resistance status in Anopheles gambiae in southern Benin. Malar J 2010, 9:83.

9. Trape J-F, Tall A, Diagne N, Ndiath O, Ly AB, Faye J, Dieye-Ba F, Roucher C, Bouganali C, Badiane A, Sarr FD, Mazenot C, Toure-Balde A, Raoult D, Druilhe P, Mercereau-Puijalon O, Rogier C, Sokhna C: Malaria morbidity and pyrethroid resistance after the introduction of insecticide-treated bednets and artemisinin-based combination therapies: a longitudinal study. Lancet Infect Dis 2011, 11:925-932.

10. Griffin JT, Hollingsworth TD, Okell LC, Churcher TS, White M, Hinsley W, Bousema T, Drakeley CJ, Ferguson NM, Basanez M-G, Ghani AC: Reducing Plasmodium falciparum malaria transmission in Africa: a model-based evaluation of intervention strategies. PLoS Med 2010, 7:e1000324.

11. Blanford S, Chan BHK, Jenkins N, Sim D, Turner RJ, Read AF, Thomas MB: Fungal pathogen reduces potential for malaria transmission. Science 2005, 308:1638-1641.

12. Farenhorst M, Farina D, Scholte EJ, Takken W, Hunt RH, Coetzee M, Knols BGJ: African water storage pots for the delivery of the entomopathogenic fungus Metarhizium anisopliae to the malaria vectors Anopheles gambiae s.s. and Anopheles funestus. Am J Trop Med Hyg 2008, 78:910-916.

13. Lwetoijera DW, Sumaye RD, Madumla EP, Kavishe DR, Mnyone LL, Russell TL, Okumu FO: An extra-domiciliary method of delivering entomopathogenic fungus, Metharizium anisopliae IP 46 for controlling adult populations of the malaria vector. Anopheles arabiensis. Parasit Vectors 2010, 3:18.

14. Mnyone LL, Kirby MJ, Lwetoijera DW, Mpingwa MW, Knols BGJ, Takken W, Russell TL: Infection of the malaria mosquito, Anopheles gambiae, with two species of entomopathogenic fungi: effects of concentration, coformulation, exposure time and persistence. Malar J 2009, 8:309.

15. Scholte EJ, Ng'habi K, Kihonda J, Takken W, Paaijmans K, Abdulla S, Killeen GF, Knols BGJ: An entomopathogenic fungus for control of adult African malaria mosquitoes. Science 2005, 308:1641-1642.

16. Farenhorst M, Knols BGJ, Thomas MB, Howard AFV, Takken W, Rowland M, N'Guessan R: Synergy in efficacy of fungal entomopathogens and permethrin against West African insecticide-resistant Anopheles gambiae mosquitoes. PloS One 2010, 5:e12081.

17. Farenhorst M, Mouatcho JC, Kikankie CK, Brooke BD, Hunt RH, Thomas MB, Koekemoer LL, Knols BGJ, Coetzee M: Fungal infection counters insecticide resistance in African malaria mosquitoes. Proc Natl Acad Sci USA 2009, 106:17443-17447.

18. Kikankie CK, Brooke BD, Knols BGJ, Koekemoer LL, Farenhorst M, Hunt RH, Thomas MB, Coetzee M: The infectivity of the entomopathogenic fungus Beauveria bassiana to insecticide-resistant and susceptible Anopheles arabiensis mosquitoes at two different temperatures. Malar J 2010, 9:71.
19. Koenraadt CJM, Takken W: Viability of GM fungi crucial to malaria control. Science 2011, 332:175.

20. Lines JD, Myamba J, Curtis CF: Experimental hut trials of permethrinimpregnated mosquito nets and eave curtains against malaria vectors in Tanzania. Med Vet Entomol 1987, 1:37-51.

21. Njie M, Dilger $E$, Lindsay SW, Kirby MJ: Importance of eaves to house entry by anopheline, but not culicine, mosquitoes. J Med Entomol 2009, 46:505-510.

22. Gillies MT: Studies on house leaving and outside resting of Anopheles gambiae Giles and Anopheles funestus Giles in East Africa. II. The ecodus from houses and the house resting population. Bull Entomol Res.

23. Luz C, Mnyone LL, Sangusangu R, Lyimo IN, Rocha LFN, Humber RA, Russell TL: A new resting trap to sample fungus-infected mosquitoes, and the pathogenicity of Lecanicillium muscarium to culicid adults. Acto Trop 2010, 116:105-107.

24. Hughes WO, Petersen KS, Ugelvig LV, Pedersen D, Thomsen L, Poulsen M Boomsma JJ: Density-dependence and within-host competition in a semelparous parasite of leaf-cutting ants. BMC Evol Biol 2004, 4:45.

25. Vandenberg JD, Ramos M, Altre JA: Dose-response and age- and temperature-related susceptibility of the diamondback moth (Lepidoptera: Plutellidae) to two isolates of Beauveria bassiana (Hyphomycetes: Moniliaceae). Environ Entomol 1998, 27:1017-1021.

26. Charlwood JD, Smith T, Kihonda J, Heiz B, Billingsley PF, Takken W: Densityindependent feeding success of malaria vectors (Diptera, Culicidae) in Tanzania. Bull Entomol Res 1995, 85:29-35.

27. Okumu FO, Killeen GF, Ogoma S, Biswaro L, Smallegange RC, Mbeyela E, Titus E, Munk C, Ngonyani H, Takken W, Mshinda H, Mukabana WR, Moore SJ: Development and field evaluation of a synthetic mosquito lure that is more attractive than humans. PLoS One 2010, 5:e8951.

28. Russell TL, Lwetoijera DW, Maliti D, Chipwaza B, Kihonda J, Charlwood JD, Smith TA, Lengeler C, Mwanyangala MA, Nathan R, Knols BGJ, Takken W, Killeen GF: Impact of promoting longer-lasting insecticide treatment of bed nets upon malaria transmission in a rural Tanzanian setting with pre-existing high coverage of untreated nets. Malar J 2010, 9:187.

29. Mnyone LL, Russell TL, Lyimo IN, Lwetoijera DW, Kirby MJ, Luz C: First report of Metarhizium anisopliae IP 46 pathogenicity in adult Anopheles gambiae s.s. and An. arabiensis (Diptera; Culicidae). Parasit Vectors 2009, 2:59.

30. Farenhorst M, Knols BGJ: A novel method for standardized application of fungal spore coatings for mosquito exposure bioassays. Malar J 2010, 9:27.

31. Ogoma SB, Lwetoijera DW, Ngonyani H, Furer B, Russell TL, Mukabana WR, Killeen GF, Moore SJ: Screening mosquito house entry points as a potential method for integrated control of endophagic filariasis, arbovirus and malaria vectors. PLoS Neglect Trop D 2010, 4:e773.

32. Smith A, Hudson JE: A modification to an experimental hut to reduce mosquito eaves-egress., WHO/Mal/72.775 1972:6.

33. Hancock PA, Thomas MB, Godfray HCJ: An age-structured model to evaluate the potential of novel malaria-control interventions: a case study of fungal biopesticide sprays. Proc R Soc B-Biol Sci 2009, 276:71-80.

34. Smith DL, McKenzie FE: Statics and dynamics of malaria infection in Anopheles mosquitoes. Malar J 2004, 3:13.

35. Russell TL, Govella NJ, Azizi S, Drakeley CJ, Kachur SP, Killeen GF: Increased proportions of outdoor feeding among residual malaria vector populations following increased use of insecticide-treated nets in rural Tanzania. Malar J 2011, 10:80.

36. Killeen GF, Smith TA, Ferguson HM, Mshinda H, Abdulla S, Lengeler C, Kachur SP: Preventing childhood malaria in Africa by protecting adults from mosquitoes with insecticide-treated nets. PLoS Med 2007, 4:1246-1258

37. Mahande A, Mosha F, Mahande J, Kweka E: Feeding and resting behaviour of malaria vector, Anopheles arabiensis with reference to zooprophylaxis. Malar J 2007, 6.

38. Howard AFV, Koenraadt CJM, Farenhorst M, Knols BGJ, Takken W: Pyrethroid resistance in Anopheles gambiae leads to increased susceptibility to the entomopathogenic fungi Metarhizium anisopliae and Beauveria bassiana. Malar J 2010, 9:168.

39. Mnyone LL, Kirby MJ, Lwetoijera DW, Mpingwa MW, Simfukwe ET, Knols BGJ, Takken W, Russell TL: Tools for delivering entomopathogenic fungi to malaria mosquitoes: effects of delivery surfaces on fungal efficacy and persistence. Malar J 2010, 9:246. 
40. Bogus Ml, Kedra E, Bania J, Szczepanik M, Czygier M, Jablonski P, Pasztaleniec A, Samborski J, Mazgajska J, Polanowski A: Different defense strategies of Dendrolimus pini, Galleria mellonella, and Calliphora vicina against fungal infection. J Insect Physiol 2007, 53:909-922.

41. Hancock PA: Combining fungal biopesticides and insecticide-treated bednets to enhance malaria control. PLoS Comput Biol 2009, 5:e1000525.

42. Reddy MR, Overgaard HJ, Abaga S, Reddy VP, Caccone A, Kiszewski AE, Slotman MA: Outdoor host seeking behaviour of Anopheles gambiae mosquitoes following initiation of malaria vector control on Bioko Island. Equatorial Guinea. Malar J 2011, 10:184.

43. Govella NJ, Okumu FO, Killeen GF: Short Report: insecticide-treated nets can reduce malaria transmission by mosquitoes which feed outdoors. Am J Trop Med Hyg 2010, 82:415-419.

44. Mnyone LL, Koenraadt CJM, Lyimo IN, Mpingwa MW, Takken W, Russell TL: Anopheline and culicine mosquitoes are not repelled by surfaces treated with the entomopathogenic fungi Metarhizium anisopliae and Beauveria bassiana. Parasit Vectors 2010, 3:80.

45. Blanford S, Shi WP, Christian R, Marden JH, Koekemoer LL, Brooke BD, Coetzee M, Read AF, Thomas MB: Lethal and pre-lethal effects of a fungal biopesticide contribute to substantial and rapid control of malaria vectors. PLoS One 2011, 6:e23591.

46. Thomas MB, Read AF: Can fungal biopesticides control malaria? Nat Rev Microbiol 2007, 5:377-383.

doi:10.1186/1475-2875-11-87

Cite this article as: Mnyone et al:: Exploiting the behaviour of wild

malaria vectors to achieve high infection with fungal biocontrol agents. Malaria Journal 2012 11:87.

\section{Submit your next manuscript to BioMed Central and take full advantage of:}

- Convenient online submission

- Thorough peer review

- No space constraints or color figure charges

- Immediate publication on acceptance

- Inclusion in PubMed, CAS, Scopus and Google Scholar

- Research which is freely available for redistribution

Submit your manuscript at www.biomedcentral.com/submit 